\title{
From family planning to sexual and reproductive health: policy implications
}

\author{
Lindsay Edouard
}

\section{Background}

Providers of family planning services deserve much credit for the development of the current concept of sexual and reproductive health. With their early recognition that family planning is the centrepiece of matters pertaining to reproduction and sexuality, the term 'sexual medicine' was appended whenever family planning was mentioned in the objectives of the National Association of Family Planning Doctors. The theme of their seventh annual general meeting was medical gynaecology, and articles in the British Journal of Family Planning in 1980 addressed topics such as rape, in vitro fertilisation, menstrual regulation and medico-legal problems, as well as broader issues such as demography and population policies. Whereas meagre resources were then available to address those issues, subsequent events led to the emergence of the co-ordinated discipline of sexual and reproductive health, which now has international support for service provision.

\section{Information dissemination}

As information gathering then depended largely on the printed word, the lack of an excellent book on contraception was deplored until the publication in 1979 of an excellent textbook "with a forbidding title and a frightening price ... seemed to promise all things to all men". ${ }^{1}$ Its "clear authoritative guidance" was welcomed, although the authors "show no reluctance to state their own opinions, always supporting them with reasoned argument".

The Journal of Family Planning Doctors was first published in 1975 and its rapidly increasing importance over the following 2 years led to its upgrading to the British Journal of Family Planning, the title used until the adoption of the current name of the Journal in 2001. The publication received crucial support, both financial and technical,2,3 from a major drug company until 1980 when the publishers changed from that drug company to Professional and Scientific Publications of the British Medical Association, additional financial resources being obtained through several advertisers.

\section{Initiating services}

Credit should be given to non-governmental organisations for their pioneering efforts, for both advocacy and service delivery, in family planning. ${ }^{4}$ In those days, the clinical management of sexuality issues was not deemed to be within the professional remit of medical practice. Therefore, dedicated family planning clinics assumed this role until the changing professional stances that led to family planning becoming an integral part of wellorganised family medicine. ${ }^{5}$

Opportunistic approaches were used to address unmet

J Fam Plann Reprod Health Care 2005; 31(2): 168-169

Larchmont, USA

Lindsay Edouard, FRCOG, MFFP, Member of the Editorial Advisory Board

Correspondence to: Professor Lindsay Edouard.

E-mail: soranae@gmail.com need by increasing access to and uptake of services. Targeted at hard-to-reach individuals, domiciliary family planning services were viewed as bridging the gap between medical and social services whilst also being of value to detect gender-based violence. ${ }^{6}$ Meanwhile, consultations in family planning clinics addressed a wide range of sexuality problems from adolescence through religion and culture to the menopause. ${ }^{7}$

\section{Policy formulation}

Demographic perspectives were still prominent around 1980 with the perceived "tie-up between birth control and death control"4 and the view that "medical practitioners have been one of the main agents in triggering off the population explosion" 1 besides concern for the hazard of pollution from increased consumption of energy. ${ }^{8}$ Couples seeking vasectomy services were labelled as "escapers" when they "felt that they should not procreate because the world situation is too threatening and the population explosion must be curbed". 9

However, soon after, the demographic perspective would give way to an emphasis on personal choice as an essential element of the libertarian aspect of health care delivery as applied to sexual and reproductive health. Contraceptive service provision would subsequently use a 'cafeteria' approach with informed choice, from a range of safe and effective methods, to suit the perceived needs of the individual. The Programme of Action of the International Conference on Population and Development, that was held in 1994 in Cairo, stressed the goal of achieving universal access to those services by 2015.10

All too often, resources for sexual and reproductive health services are still being directed towards the development of excellent policy statements, which are not subsequently implemented in service delivery. The selection of effective interventions is crucial but the availability of trained staff is often a limiting factor. In areas such as emergency contraception where there are sensitive client-provider interactions, services are often provided without any formal policy statement as the policy formulation process would lead to official discussions which might jeopardise the service.

\section{From service integration to co-ordination}

Integration of services for family planning, maternal health and sexually transmitted infections (STIs) (including HIV) - the three central components of reproductive health poses major challenges especially in resource-poor situations. More evidence is needed to demonstrate that integration leads to improved quality of care as exemplified by the incorporation of certain tasks for the control of STIs within a family planning consultation. ${ }^{11}$

In resource-poor situations, the same individual often performs most tasks related to reproductive health and integration occurs inevitably. In such cases, co-ordination is necessary at the higher level in the organisation of services. The importance of the private sector in service delivery should not be underestimated, especially with the increasing role of independent practitioners in the provision of primary health care services in resource-poor countries. 


\section{The opportunity}

International conferences of the 1990s led to an emphasis on reproductive health, which is at the intersection of women's health, human rights and environmental issues. Adopted by the General Assembly of the United Nations in September 2000, the Millennium Declaration ${ }^{12}$ led to an initiative which is fully supported by numerous funding bodies and aimed at achieving eight millennium development goals. Three of these goals are directly related to reproductive health (reductions in maternal and child mortality besides control of the spread of HIV), whereas the other five are complementary to reproductive health: gender equality, primary education, environmental sustainability, poverty reduction and partnership for development.

As human rights are being increasingly defined in operational terms to match available resources, public health aspects of reproductive rights should be applied in health service reform. Poverty reduction strategies and sector-wide approaches are being promoted in developing countries for resource mobilisation. Strategic directions should emphasise an evidence-based approach not only for service delivery but also for policy formulation, demand creation and advocacy to avoid the ineffective use of resources.

The editorial independence of professional journals is vital for encouraging debates on controversial issues. The recent setting-up of registers for drug trials will ensure the prompt availability of information, which should be discussed in journals. This Journal is the official publication of the Faculty, a specialty-based body without any political or geographical affiliation, and it operates with a fully independent mandate unlike some other, apparently comparable, journals which have faced major problems over disputes with their umbrella sponsors recently. The independent stance of this Journal was carefully protected at its inception and it is our duty to disseminate objective information to further comprehensive reproductive health services.

Statements on funding and competing interests

Funding. None identified.

Competing interests. None identified.

References

1 Loudon N. Human fertility control: theory and practice. Br J Fam Plann 1980; 5: 101-102.

Editorial. Thanks to Wyeth. Br J Fam Plann 1980; 5: 99

3 Melville B, Wagstaff E. Annual meeting of affiliated group representatives with NAFPD Council. Br J Fam Plann 1980; 6: $27-28$.

4 Jackson M. The changing face of family planning. Br J Fam Plann 1980; 6: 71-74.

5 Upsdell $\mathrm{M}$. The timing and extent of examinations of women using oral contraception - a discussion. Br J Fam Plann 1980; 6: 42-45.

6 Christopher E. Domiciliary family planning - a service described and assessed. Br J Fam Plann 1980; 6: 3-9.

7 Anonymous. In the clinic. Br J Fam Plann 1980; 6: 94-95.

8 Morris G. Global signposts to the 21st century. Br J Fam Plann 1980; 5: 102 .

9 Thexton R, Lincoln R. The Institute of Psycho-sexual Medicine: report. Br J Fam Plann 1980; 5: 103-104.

10 Guillebaud J. After Cairo. Br J Obstet Gynaecol 1995; 102: 436-438.

11 Dehne K, Snow R. Integrating STI Management into Family Planning Services: What are the Benefits? Document WHO/RHR/99.10. Geneva, Switzerland: World Health Organization, 1999; 61-62.

12 Grown C, Gupta GR, Pande R. Taking action to improve women's health through gender equality and women's empowerment. Lancet 2005; 365: 541-543.

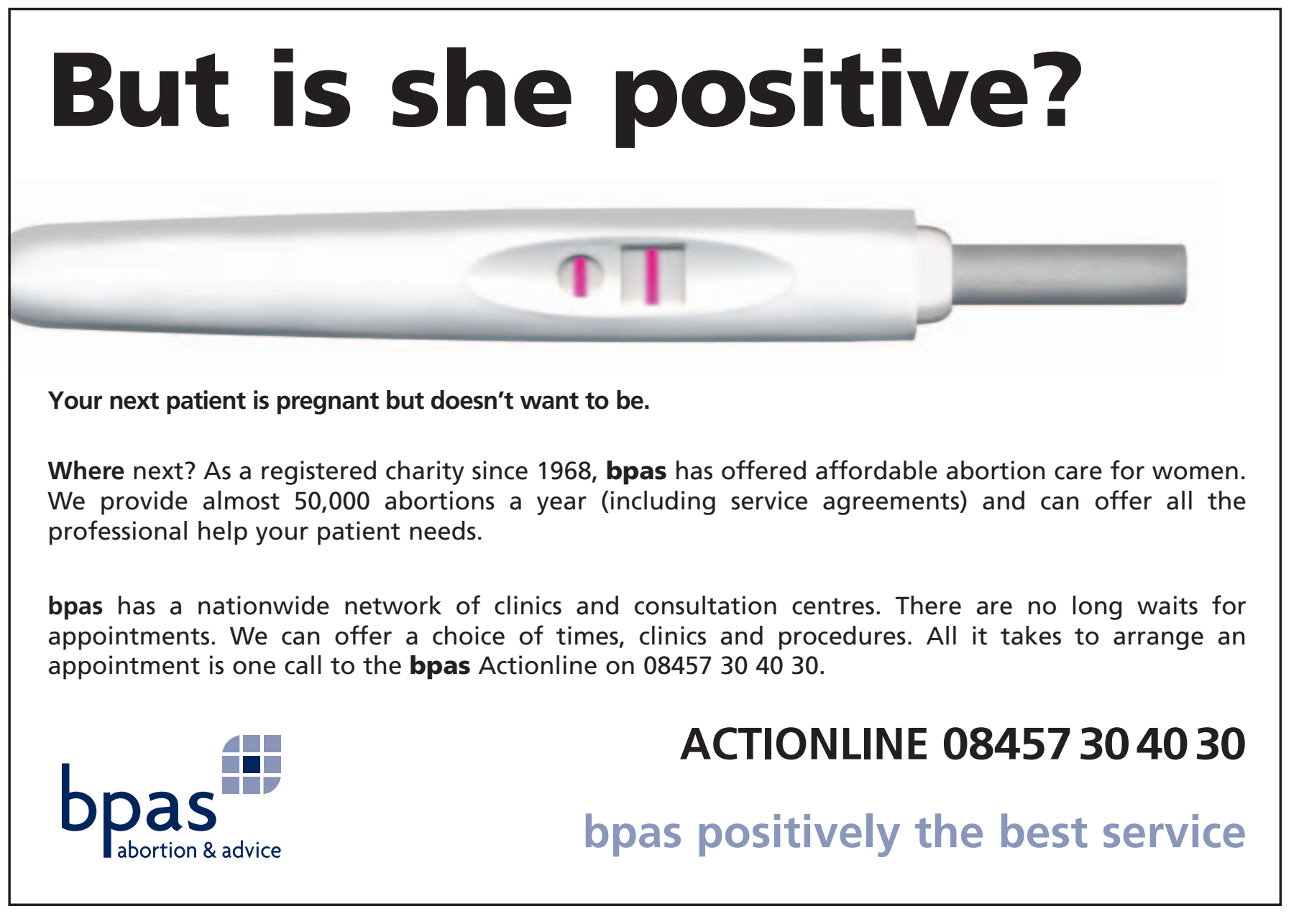

